\title{
Bone histological correlates of soaring and high-frequency flapping flight in the furculae of birds
}

Jessica Mitchell $^{1 *}$, Lucas J. Legendre ${ }^{2}$, Christine Lefèvre ${ }^{3}$, Jorge Cubo ${ }^{4}$

${ }^{1}$ Steinmann-Institut für Geologie, Mineralogie und Paläontologie, Universität Bonn, Nussallee 8, D-53115 Bonn, Germany

${ }^{2}$ Department of Karoo Palaeontology, National Museum, 36, Aliwal Street, Bloemfontein, 9301, South Africa

${ }^{3}$ MNHN/CNRS/Sorbonne Universités, Archéozoologie et Archéobotanique: sociétés, pratiques et environnements (UMR 7209), CP 55, 55 rue Buffon, F-75005 Paris, France

${ }^{4}$ Sorbonne Universités, UPMC-Univ. Paris 06, CNRS, Institut des Sciences de la Terre de Paris (ISTeP), 4 place Jussieu, BC 19, F-75005 Paris, France

* Corresponding author.

Email: mitchell20j@gmail.com

\section{Highlights}

- Furculae of soaring birds have increased Haversian bone density.

- High-frequency flapping flight is not different from low-frequency flapping.

- Depression forces and size increase in soaring birds may explain Haversian bone increase. 


\begin{abstract}
The furcula is a specialized bone in birds involved in flight function. Its morphology has been shown to reflect different flight styles from soaring/gliding birds, subaqueous flight to high-frequency flapping flyers. The strain experienced by furculae can vary depending on flight type. Bone remodeling is a response to damage incurred from different strain magnitudes and types. In this study, we tested whether a bone microstructural feature, namely Haversian bone density, differs in birds with different flight styles, and reassessed previous work using phylogenetic comparative methods that assume an evolutionary model with additional taxa. We show that soaring birds have higher Haversian bone densities than birds with a flapping style of flight. This result is probably linked to the fact that the furculae of soaring birds provide less protraction force and more depression force than furculae of birds showing other kinds of flight. The whole bone area is another explanatory factor, which confirms the fact that size is an important consideration in Haversian bone development. All birds, however, display Haversian bone development in their furculae, and other factors like age could be affecting the response of Haversian bone development.
\end{abstract}

Keywords: Furcula; Haversian bone tissue; Phylogenetic generalized least squares; Phylogenetic comparative methods; Bird flight 


\section{Introduction}

The furcula is an essential part of the skeletal adaptations for flight in birds (Close and Rayfield, 2012).

Originally thought to have a static function as a spacer of the pectoral girdle and origin of the flight muscles, the furcula has been shown to play a dynamic role during flight, undergoing extreme deformations (Jenkins et al., 1988). According to these authors, the deformation during the wing beat cycle could allow for inflation and deflation of the interclavicular air sac (Jenkins et al., 1988). More recently, Hui (2002) showed that the shape of the furcula is more correlated to the type of flight than to taxonomy. Hui categorized birds into 'soaring with no flapping', 'flapping with no soaring', 'subaqueous' (i.e., all wing beats taking place under water) or 'partial subaqueous' (i.e., wing beats used for both aerial and submerged flapping) and found that soaring birds share a very specialized, flat and U-shaped furcula providing less protraction force and more depression force than the furcula of birds showing other kinds of flight (Hui, 2002). This was further supported by the more extensive study of Close and Rayfield (2012), who found similar results, with soaring birds having the 'U'-shaped furcula. They found a low but significant correlation between furcula shape and phylogeny, but the results of their phylogenetic flexible discriminant analysis had a higher rate of misclassification than Hui (2002) (40\% misclassification in Close and Rayfield, 2012 compared to 25\% in Hui, 2002). They noted that Hui (2002) may have overstated the findings on the strength of the relationship between form and function. Nonetheless, on the morphological level, some correspondence with form and function is apparent. Because the morphological specialization of the furcula of soaring birds involves less protraction and more depression forces than in birds showing other kinds of flight (Hui, 2002), a first aim of the present study involves testing whether this functional difference is reflected in the bone microstructure.

It has been shown that the effect of cyclic deformations, which would occur in the furcula during flapping flight, is reflected on the histological level. Bone remodeling has been shown to be associated with fatigue and microcrack damage (Burr et al., 1985; Mori and Burr, 1993; Lee et al., 2002; Cardoso et al., 2009), suggesting that repetitive loading stimulates the removal and replacement of damaged bone (replacement bone $=$ Haversian bone). In the cortex, damaged bone is replaced by secondary osteons, which are formed by the coupled action of osteoclasts (removal of bone) and osteoblasts (deposition of centripetally infilling bone lamellae) in small discrete packets (Parfitt, 1994).

Static tensile strains of 0.004 (Reilly and Currey, 1999) and cyclic tensile strains of 0.014-0.015 (Burr et al., 1985) have been shown to produce microcracks. Considering that the furcula is exposed to strain levels of 0.007-0.151 (Bailey and DeMont, 1991), we expect to find microcracks and/or secondary osteons (the outcome of microcrack reparation). Moreover, in some bones of certain vertebrates, including the furcula of birds, Haversian bone tends to be localized in certain areas, which likely correspond to areas under higher strain. For instance, Mayya et al. (2016) found that Haversian tissue formed only in regions under compressive strain in bovine femora, whereas regions withstanding tensile strains remained as primary tissue (Mayya et al., 2013). 
In a previous study, Ponton et al. (2007) performed a comparative analysis to test the effect of high- versus lowfrequency flapping flight on Haversian bone density (HBD) using the variation partitioning method and a sample of 29 species of birds. The outcome of the analysis indicated that a large percentage of variation of Haversian bone density was explained by body mass, type of flight and phylogeny (Ponton et al., 2007). Birds that were categorized as having higher frequency flapping flight had significantly more Haversian bone than those categorized as having other types of flight of lower wing beat frequency. It was also considered that phylogenetic signal in Haversian bone density may have been a by-product of phylogenetic signal in body mass or type of flight (Ponton et al., 2007). However, the phylogenetic eigenvector regressions the analyses of Ponton et al. (2007) are based on have been criticized because they do not assume an evolutionary model (Adams and Church, 2011; Freckleton et al., 2011; but see also Diniz-Filho et al., 2012, who showed that phylogenetic eigenvector regressions perform well when an appropriate phylogenetic eigenvector selection procedure is used). A second aim of the present study thus involves testing the hypothesis of Ponton et al. (2007) by re-analyzing the original data and using new methods that explicitly assume an evolutionary model - namely phylogenetic generalized least squares (PGLS; Grafen, 1989) and phylogenetic analysis of variance (phyANOVA; Garland et al., 1999), as well as an updated phylogeny (for both topology and divergence times) and a more comprehensive sample.

\section{Materials and methods}

\subsection{Materials}

We used a sample of furculae of 55 specimens belonging to 41 species of birds (see Table 1 for a complete list and Fig. 1 for the phylogeny). This sample also included the sections analyzed by Ponton et al. (2007). Several of the new, additional species include birds with a soaring/gliding flight type, which was relatively underrepresented in the study by Ponton et al. (2007). We analyzed the midshaft cross-sectional area of one of the furcula branches (the sections of Ponton et al., 2007 were re-analyzed, so that all sections were analyzed by the same scientist (J.M.) to minimize measurement error). The specimens are all from the Muséum National d'Histoire Naturelle and the Université P. \& M. Curie in Paris.

Following Viscor and Fuster (1987), bird species were assigned to different flight types based on the frequency of flapping. Birds categorized as high-frequency flappers (type 1 in Table 1) are considered to have an elevated respiration frequency to wing beat ratio of 4-5:1, with strong pectoral muscles (Viscor and Fuster, 1987). Birds, particularly cursorial birds with rapid take-offs and short flights (type 2 in Table 1) are adapted to short bursts with short broad wings (Viscor and Fuster, 1987). Birds considered having an undulating flight (type 3 in Table 1) have periods of soaring flight with intermittent powered flight. Soaring/gliding birds (type 4 in Table 1) have large wing areas and sustain long flights without flapping (Viscor and Fuster, 1987). Finally, forward flapping birds (type 5 in Table 1) use a sustained horizontal flight, including the bounding flight style of many passerine birds (Viscor and Fuster, 1987).

In addition to considering the Viscor and Fuster categories, we also used binary categories to group birds for analysis. In one dataset, soaring/gliding versus flapping, all birds not in the soaring/gliding category were grouped in the flapping category (soaring vs. flapping). In another dataset, following Ponton et al. (2007), we compared high- versus low-frequency flapping where birds classified in the high-frequency flapping category of Viscor and Fuster (1987) were put in one category and all other birds were placed in a second category of all other wing beat frequencies (high vs. low frequency). Although birds belonging to the forward flapping type can have relatively high-frequency flapping, the categorization as used by Viscor and Fuster (1987) is implemented 
here for consistency with the study by Ponton et al. (2007).

Viscor and Fuster (1987), Hui (2002) and Close and Rayfield (2012) have all classified birds based on flight type, although with some discrepancies. We chose to use Viscor and Fuster's classification because their dataset includes nearly all the species we analyzed. In contrast, Hui (2002) only provides 13 species in his analysis, with only three genera having matches in our dataset. The study by Close and Rayfield (2012) shares only nine taxa with our dataset. These publications differ in their categorization of some species. Hui (2002) identified Fregata magnificens and Fregata minor as soaring birds, while the species in our dataset, Fregata aquila, was classified as undulating flyer in Viscor and Fuster (1987). Hui (2002) identified Uria aalge as partially subaqueous, while Viscor and Fuster (1987) classified them as high-frequency flappers (they had no sub-aqueous category). Finally, Columba livia was categorized as flapping by Hui (2002), and Columba palumbus was categorized as sustained forward flapping by Viscor and Fuster (1987).

Close and Rayfield (2012) studied several taxa that are also included in Viscor and Fuster (1987). In general, species classified as 'continuously flapping' by Close and Rayfield (2012) are identified as birds with high-frequency flapping, sustained forward flapping, or undulating flight by Viscor and Fuster (1987). The soaring category of Close and Rayfield (2012) typically coincided with the undulating flight of Viscor and Fuster (1987), but there were only a few comparable taxa in the datasets. Larus is an exception and is considered soaring/-gliding by Viscor and Fuster (1987).

\subsection{Biometry}

Photographs of the furculae were taken with a Leica DMLP compound microscope and Leica DFC420 camera attachment (Leica Microsystems, Wetzlar, Germany). We also made descriptions of the histology and microanatomy of the furculae. The complete cross-sectional area (whole bone area, WBA, $\mathrm{mm}^{2}$ ), cortical area (i.e., the complete cross-sectional area minus the medullary area; $\mathrm{CA}, \mathrm{mm}^{2}$ ) and Haversian bone area (HBA, $\mathrm{mm}^{2}$ ) were measured using the image processing software Fiji (http://fiji.sc/Fiji). In cases where multiple specimens were available for a single species, the average of all values is reported as well as the standard deviation (Table 1). Haversian bone density (HBD) was determined by the ratio of HBA to CA. Ponton et al. (2007) also obtained the HBD using the ratio of HBA and CA, but many of the resulting Haversian bone densities obtained in the present study differ from those in Ponton et al. (2007). This may be due to the medullary area being measured differently. In the present study, when a specimen contained two or more medullary regions separated by bony struts, only the empty space was counted and the bone strut was not included (Fig. 2).

\subsection{Phylogenetic comparative methods}

In statistical analyses, there are several assumptions made about the data, such as normality, homoscedasticity and independence of observations. When comparing traits of different species, independence is considered potentially violated because of the evolutionary relationships of species to each other; thus, phylogeny needs to be considered as part of the analysis (Felsenstein, 1985). A phylogenetic tree of the clade Aves was implemented in the present analysis based on the topology and branch lengths of Prum et al. (2015), with topologies for the inner clades Charadriiformes and Passeriformes being based on Thomas et al. (2004) and Jonsson and Fjeldsa (2006), respectively (Fig. 1). Although Haversian bone density may largely be a response to the mechanical loading environment (Burr et al., 1985; Ericksen, 1991; Lee et al., 2002; Mayya et al., 2016) and not a character 
dependent on relatedness of species, it is strongly recommended that comparative data be corrected for phylogenetic dependence regardless (Freckleton et al., 2002). Moreover, causal (explanatory) variables of HBD could contain a significant phylogenetic signal, in which case the use of phylogenetic comparative methods is mandatory.

PGLS (Grafen, 1989) and phyANOVA (Garland et al., 1999) are both very well-known and frequently used phylogenetic comparative methods (see Garamszegi, 2014 for an extensive review). Both procedures are designed to take into account the influence of evolutionary relationships between species (i.e., phylogenetic signal sensu Blomberg and Garland, 2002) in a generalized linear model, whether in a regression of one or more continuous predictors over a response variable (PGLS) or in an analysis of variance of one or more discrete factors over a response variable (phyANOVA).

Since the evolutionary model that describes the evolutionary path of HBD for our dataset is not known, we first compared several models and determined their goodness of fit based on their AICc score, i.e., the Akaike information criterion corrected for finite sample size (Burnham et al., 2011). Using the fitContinuous function in the R package 'geiger' (Harmon et al., 2008), we were able to compare AICc scores for four different evolutionary models: Brownian Motion (BM), i.e., a purely neutral model of evolution (Felsenstein, 1973); Ornstein-Uhlenbeck (OU), i.e., a model with selective optima along the branches (Butler and King, 2004); Early Burst (EB), i.e., a model with exponential increases and decreases of evolutionary rate through time (Harmon et al., 2010); and White Noise, i.e., no phylogenetic effect, assuming a single normal distribution of the data. When assuming no measurement error, the model with the lowest AICc score was the OU model; however, with consideration of measurement error, the BM model had the lowest AICc score. Thus, we used BM as the evolutionary model to analyze the data.

PGLS and phyANOVA do not require a given dataset to be normally distributed and homoscedastic prior to analysis, but after, since residuals corrected for phylogenetic non-independence are assumed to follow the same distribution as any non-phylogenetically influenced dataset (Freckleton, 2009). Both procedures, however, have been used frequently to analyze comparative datasets in many studies, often assuming the data to be normally distributed without properly testing it in the residuals. What effect non-normal data has on PGLS results has not been well investigated (Mundry, 2014), but since it is possible to test this, it should be considered. We used the Lilliefors test from the R package 'nortest' (Gross and Ligges, 2015; https://CRAN.Rproject.org/package $=$ nortest) to test for normality, and plotted the data as a Q-Q plot and compared residual and fitted data points to visually see how our data fit with the assumptions of normality and homoscedasticity (Fig. 3). The Q-Q plot and fitted vs normalized residual plots can be useful in determining if there are outliers or problematic taxa that may be skewing the distribution (Mundry, 2014). In our case, our initial Q-Q plot was skewed, and residuals corrected for phylogenetic non-independence failed to pass for normality. To obtain normal distribution, we had to remove six taxa from the original dataset (see Fig. 3 and Table 1). We ran PGLS using the function pgls of the package 'caper' (Orme et al., 2013), and phyANOVA using the function gls of the package 'nmle' (Pinheiro et al., 2016) and the phylogenetic correlation structure corPagel (for the BM model) specified in the package 'ape' (Paradis et al., 2004), in R (R Core Team, 2015). We used HBD as our dependent variable and used PGLS to test if WBA (a proxy for size) was a trait that influenced HBD. Three models using PGLS were tested: a simple linear regression with one predictor (WBA or one of the flight categories); the additive model, which tests if a change in HBD is correlated to a change in flight mode (e.g., from soaring to flapping) at a given size of WBA and vice versa (i.e., a change in HBD is correlated to a change 
in WBA at a given flight mode); and the interaction model, which tests if the relationship between WBA and HBD is different for each flight mode and vice versa.

For the phyANOVA, we categorized the dataset based on Viscor and Fuster (1987) as soaring versus flapping flight, and high versus low frequency of flapping flight, to test the influence of flight type on HBD. In addition to our dataset, we also re-tested the Ponton et al. (2007) dataset using phyANOVA along with the updated phylogeny and divergence times to see how the variation partitioning method compares to the new analysis. Finally, we tested phylogenetic signal on HBD and WBA using Blomberg's $K$ and Pagel's lambda (Münkemüller et al., 2012), both assuming a Brownian motion model.

\section{Results}

\subsection{Simple linear regression and phyANOVA results}

A significant relationship existed between HBD and WBA as well as HBD and soaring vs. flapping flight (Fig.

4). Other bivariate tests were not significant. Eleven percent of the total variability in HBD was explained by WBA. Twenty-seven percent of the total variability in HBD was explained by soaring vs. flapping flight. In the last box plot of Fig. 4, type 4 flight (corresponding to soaring/gliding flight) stands out among the other flight types. PhyANOVA showed no difference between the high-frequency flapping flight versus other types of flight of lower wing beat frequency, but a difference was observed in the soaring versus flapping flight. The phyANOVA was strongly significant $(F$-value $=12.40382, p=0.0013)$, which means that soaring vs. flapping flight is useful for predicting HBD (see Tables 2 and 3).

\subsection{Additive model results}

WBA is predictive of HBD in the models that include high- vs. low-frequency flapping flight and type of flight, but not with soaring vs. flapping. In the latter case, soaring vs. flapping is a good predictor of HBD. For a change from soaring flight to flapping flight (the values change from 0 to 1 ), the model predicts that HBD decreases by -0.140179 when holding WBA constant. Twenty-five percent of the total variability in HBD is explained by soaring vs. flapping flight. The model overall is useful for predicting $\operatorname{HBD}(F=6.566, p=0.00408)$ (see Table 2).

\section{Interaction model results}

None of the results showed significance except WBA in the model with high- vs. low-frequency flapping flight. Although in other tests soaring vs. flapping flight has been a significant predictor, it is not in this model, which means if HBD increases (or decreases), the size of WBA is not dependent on whether the flight mode is gliding or soaring. However, the overall model is significant according to the $F$ test $(F=4.35, p=0.01141)$. This indicates the overall model is a better fit than an intercept-only model (null hypothesis), meaning the coefficients are collectively not all equal to zero. This is also true for the other two models using high- vs. low-frequency flapping and type of flight (see Table 2).

\subsection{Re-analysis of the Ponton et al. (2007) dataset}

We re-tested the effect high-frequency flapping flight versus all other types of flight of lower wing beat frequency had on HBD using the method of phyANOVA (which assumes an evolutionary model) and our 
updated phylogeny, on the original dataset of Ponton et al. (2007) and found that the results were similar to those of Ponton et al. (2007) with a highly significant $p$-value: DF: $26 ; F$-value $=8.24453 ; p$-value $=0.008$.

\subsection{Phylogenetic signal}

Variation in WBA contains a highly significant phylogenetic signal (Pagel's lambda $=0.9641092, p=$ $1.580644 \mathrm{e}-05$; Blomberg's $K=0.9576611, p=0.001)$ whereas the phylogenetic signal contained in the variation in HBD is lower, still significant using Blomberg's $K(K=0.4511969, p=0.035)$ but not significant using Pagel's lambda (lambda $=0.4026081, p=0.1655004)$.

\subsection{Histological and microanatomical description}

The furculae across the different species were relatively consistent histologically, even throughout a wide range of sizes. Most bones were lined with an inner circumferential layer (ICL) (endosteal lamellae) and an outer circumferential layer (OCL). In between, secondary osteons or primary vascularized tissue filled the cortex. The primary tissue was fibrolamellar with longitudinal canals, with some reticular canals observed occasionally, surrounded by primary osteons. Secondary osteons either filled the entire space between the OCL and ICL or were restricted to a region of the section. Orientation of the sections was not known, so it cannot be said if secondary osteons formed consistently in the same region (e.g., anterior, posterior). If thickness of the cortex varied, secondary osteons were preferentially found in the thicker regions. Osteocyte lacunae of secondary osteons were mostly fusiform. The OCL and ICL usually had quite thin osteocyte lacunae. The primary tissue varied with woven tissue having plump, densely packed lacunae and the primary osteons having similarly shaped osteocytes to secondary osteons (i.e., fusiform or spindle-shaped).

Secondary osteon size reflected, in general, the size of the specimen. The smallest birds had the smallest secondary osteons (ranging from 70 to 120 micrometers in diameter) and the largest birds had larger secondary osteons (up to 200 micrometers in diameter of the minor axis). Most secondary osteons appeared normal, although many were more elongated in the larger species (e.g., Fig. 5). Under cross-polarized light, secondary osteons were mostly 'dark', with a brighter ring around the Haversian canal. This may indicate that the collagen fibers in the lamellae were oriented more longitudinally than transversally (Ascenzi and Bonucci 1967, 1968). The microanatomy varied between species. The compactness of the section tends to decrease with increasing size, with the large birds having relatively thin cortices and a large medullary cavity, with few trabeculae and the smaller birds having small medullary cavities and thick cortices. In the center, one or several medullary cavities could be observed with an ICL surrounding each cavity.

In Fig. 5, four species are shown (sectioned at the midpoint of one of the branches) which represent the diversity in microanatomy and show the secondary osteon distribution. Emberiza and Garrulus are two species of Passeriformes and the smallest sampled specimens. Emberiza has two medullary cavities and some secondary osteons that have large canals along the right side of the image. The primary lamellar tissue on the left does not have any vascularization, which may be attributed to its small size. The ICL and OCL can also be observed. Garrulus has a different cross-sectional shape but also has two medullary cavities surrounded by an ICL. Relatively thick lamellae can be observed near the top of the image. Haversian tissue surrounds the medullary region. The larger Larus fuscus has mostly Haversian tissue in between the OCL and ICL. The secondary osteons are typically round. The cortex is quite thick, with a single medullary cavity. Vultur, the largest specimen and nearly 10 times the size of Larus, has a noticeably thin cortex relative to medullary area. However, the 
absolute cortical thickness is similar in Larus and Vultur. Despite this, the secondary osteons seem larger and much more elongated in Vultur.

\section{Discussion}

\subsection{Soaring vs. flapping flight}

The present paper has two aims. The first one is related to the findings of Hui (2002), who showed that the furculae of soaring bird species show a very specialized shape (flat, semi-circular and U-shaped) and experience less protraction force and more depression force, linked to flight function (long periods during which the wings are maintained in an extended position alternating with a few wing beats). So the first objective involves testing whether the particular function of the furculae of soaring birds (involving less protraction force and more depression force) is reflected in the extent of Haversian remodeling.

PhyANOVA analyses show that birds that use soaring flight have significantly higher HBD than birds showing other kinds of flight. This could be the outcome of either function (the semi-circular U-shaped furcula is linked to less protraction and more depression forces; Hui, 2002) or body mass. Our bivariate PGLS analyses show that both WBA (as a proxy of body mass) and the type of flight (soaring/gliding versus flapping) are significant explanatory factors of HBD, but the significance of soaring/gliding versus flapping $(p=0.0008)$ is higher than that of WBA $(p=0.0265)$. When both explanatory factors are analyzed together using a PGLS additive model, the whole model is still significant ( $p=0.004)$ but only soaring/gliding versus flapping is significant $(p=0.017)$, while WBA is not $(p=0.8041)$. Although the soaring/gliding birds represent the largest birds in the dataset, the quantitative analyses suggest that the type of flight is the driving force (rather than WBA). In the interaction model, soaring/gliding flight and WBA are not significant, which means that changes in HBD are not dependent on changes of WBA and flight mode together; in other words, there is no effect on HBD if WBA increases and flight mode changes from soaring to flapping.

It may be intuitive to assume that damage from cyclical deformations would incur higher remodeling rates. Ponton et al. (2007) showed that high-frequency flapping birds have higher Haversian bone densities than lowerfrequency flapping birds. However, the inclusion of several soaring/gliding birds in the new dataset reveals a different result. Soaring/gliding birds may increase Haversian remodeling to compensate for a relatively high damage accumulation. Greater depression forces on the U-shaped furcula of soaring/gliding birds may lead to greater microdamage, and hence trigger secondary osteon formation, compared to the greater protraction forces on the V-shaped furcula of birds that utilize flapping styles of flight. The magnitude, frequency and type of force experienced on cortical bone can affect the extent of damage and the development of secondary osteons (Robling et al., 2006). For example, studies on the femora, radii and calcanea of artiodactyls and perissodactyls have shown that regions under compressive strain tend to form more secondary osteons (Mason et al., 1995; Skedros et al., 1997; Sorenson et al., 2004; Mayya et al., 2016). An investigation of the way in which the less intensive protraction and greater depression forces by the semi-circular U-shaped furculae of soaring birds produce higher Haversian bone densities is beyond the aims of the present comparative study. Experimental analyses using strain gauges are necessary to answer this question.

Both Close and Rayfield (2012) and Hui (2002) identified soaring birds as having more U-shaped furculae. However, we used the classification of Viscor and Fuster (1987), which contains few species in common with these other studies and a few conflicting interpretations of flight type. Thus, some of the species in the soaring/gliding category in our dataset may not have a U-shaped furcula. It should be kept in mind that these 
categories are artificial and subject to the researchers' interpretations of flight styles. Close and Rayfield (2012) designated several species as 'soaring/flapping' that plot with the soaring birds, so there is some overlap between the morphology of birds of various flight types. Nonetheless, our results indicate that soaring birds have a distinct morphology, and a distinct histology, in terms of higher Haversian bone density.

\subsection{Re-analysis of the Ponton et al. (2007) dataset}

The second aim of the present study involves testing, with new methods, an updated phylogeny and a more comprehensive sample, the hypothesis by Ponton et al. (2007) suggesting that bird species characterized by highfrequency flapping flight undergo higher cyclical deformations of the furcula and develop more Haversian bone as a response to damage than bird species characterized by other types of flight of lower wing beat frequency. These new phylogenetic comparative methods (i.e., PGLS and phyANOVA), unlike the phylogenetic eigenvector regression used by Ponton et al. (2007), explicitly assume an evolutionary model. Ponton et al. (2007), using a reduced sample including fewer soaring/gliding birds and using variation partitioning based on phylogenetic eigenvector regressions, found that the type of flight (high to low frequency) was the driving force (this factor alone explained $24.1 \%$ of the variation in HBD, whereas body mass alone explained $17.7 \%$ of this variation). These results are similar to those obtained here using the same sample, phyANOVA (assuming an evolutionary model) and an updated phylogeny (DF: $26 ; F$-value $=8.24453 ; p$-value $=0.008)$.

Results obtained using the more comprehensive sample show that the bivariate PGLS regression between high to low frequency of flapping versus HBD is not significant; the additive PGLS model between high to low frequency of flapping + WBA (as a proxy of body mass) is marginally not significant $(p=0.086)$; and the interaction PGLS model is significant $(p=0.035)$. The interaction model as a whole is significantly different from the null hypothesis, which assumes the coefficients to be zero. Thus, the predictors (WBA and high- vs. low-frequency flapping) are possibly having an effect on HBD. In the case of high-to-low frequency, WBA is significant $(p=0.007762)$, which means that size is the factor that explains the change in HBD when flight mode is considered in the model. Considering that in the additive PGLS model and in the interaction PGLS model the variable high-to-low frequency of flapping is never significant whereas WBA is always significant, we conclude that this last variable (WBA) is the driving force. Phylogenetic signal on WBA (the driving force) is highly significant using both Blomberg's $K$ and Pagel's lambda, whereas phylogenetic signal on HBD is lower but still significant using Blomberg's $K$ and not significant using Pagel's lambda. We conclude that phylogenetic signal on HBD is a collateral effect of phylogenetic signal on WBA (the driving force).

The two distinct results from the original dataset of Ponton et al. (2007) and the new dataset illustrate the impact of sample selection on analyses. We maintained the same categorization of flight type to provide a more comparable analysis of the datasets. The inclusion of more species and more representatives of the soaring/gliding flight type influenced the outcome for the high- vs. low-frequency flapping analysis. Considering that the newer dataset is more comprehensive in terms of species, the significant result from the Ponton et al. (2007) dataset needs to be viewed in the context of specimens analyzed.

\subsection{Other influences on Haversian bone}

HBD has been linked with body size since it is more often observed in medium to large mammals and dinosaurs (Foote, 1916; Enlow and Brown, 1957, 1958; Currey, 2003). Currey (2003) remarked that small birds have little 
to no remodeling (presumably referring to long bones like femora and humeri), and it is the larger mammals, birds and dinosaurs that tend to extensively develop Haversian tissue. Our observations (and those of Ponton et al., 2007) indicate that even some of the smallest birds (e.g., Emberiza, mass estimate of $30 \mathrm{~g}$ ) have extensive secondary osteon development. This means that size cannot be the only factor explaining the distribution of Haversian tissue. Because the furcula is subject to deformation, it may be more prone to Haversian remodeling relative to other bones of the same specimen. If this is the case, a signal from microdamage accumulation due to flapping resulting in Haversian tissue is not evident from our results.

One other possible factor that may be influential in our analysis that we could not account for is age. Bone tends to increase in mineral content with age, and thus toughness is reduced (Currey, 2001). Haversian bone tissue also accumulates with age (Kerley, 1965; Frost, 1987), likely as a response to increased mineralization and possibly non-enzymatic collagen cross-links that degrade collagen over time (Saito and Marumo, 2010). This suggests that Haversian tissue development is related to the age of the specimen and the variation observed in the specimens may be partially due to different ages.

Another possible explanation may be related to calcium and phosphorous homeostasis. Although Parfitt (2010) suggests that remodeling for homeostasis likely occurs mostly in cancellous tissue near red marrow, this is based solely on evidence from humans and dogs. It is not known if birds meet their homeostatic needs regarding calcium with bone remodeling. In the case of egg development (Moyle and Walker, 1986) or molting (Meister, 1951; Zahnd, 1954), osteoporotic conditions have been observed in long bones of birds. Thus, it is possible that calcium is mobilized from bones during critical or stressful occasions. However, it seems unlikely that the furcula would be involved in this process because loss of bone mineral in this place may be detrimental to the flight ability of the bird.

The microanatomy varies a lot in the furculae, from high to very low compactness, and presenting different cross-sectional shapes. The thin cortex of the larger birds makes sense in that they must maintain a light skeleton. One interesting change in secondary osteons is that the larger birds have more elongated, larger secondary osteons, even though medium-sized birds like Larus have the same cortical space available to form secondary osteons. Thus, it seems that the formation of secondary osteons is responding to different dynamics in the different size classes. If loading magnitude or type dictates collagen orientation and distribution of Haversian tissue (e.g., Ascenzi and Bonucci, 1967, 1968; Skedros et al., 1996; Mayya et al., 2016), it may well also regulate the size and orientation of secondary osteons.

\section{Conclusions}

Using PGLS and phyANOVA, we found Haversian bone density to be higher in birds with soaring/gliding flight than in those with flapping flight, which differs from the re-analysis of the Ponton et al. (2007) dataset that found a significant difference between high-frequency flapping vs. all other flight types. The difference in results is largely due to the larger sample size including several soaring/gliding birds in the new dataset. The semi-circular U-shaped furcula of these soaring/gliding birds is subjected to more depression forces compared to other birds with different flight modes, which may be one of the reasons for greater Haversian bone development. In addition, the whole bone area, as a proxy for size, is also significantly correlated with Haversian bone density. Another possible factor that may influence Haversian bone density includes age, which could not be accounted for in the present study. Experimental studies on damage accumulation in the cortex of the furcula during deformation could provide insights into the microstructural patterns observed in thin sections. 


\section{Acknowledgements}

We thank the Comparative Anatomy Collections of the Paris MNHN for providing samples and Hayat Lamrous,

Paris VI University, for preparing thin sections. J.M. was funded through the Deutsche Forschungsgemeinschaft (DFG). We would also like to thank two reviewers who helped improve an earlier draft of this manuscript. 


\section{References}

Adams, D.C., Church, J.O., 2011. The evolution of large-scale body size clines in Plethodon salamanders: evidence of heat-balance or species-specific artifact? Ecography 34, 1067-1075.

Ascenzi, A., Bonucci, E., 1967. The tensile properties of single osteons. Anat. Rec. 158, 375-386.

Ascenzi, A., Bonucci, E., 1968. The compressive properties of single osteons. Anat. Rec. 161, 377-391.

Bailey, J.P., DeMont, M.E., 1991. The function of the wishbone. Can. J. Zool. 69, 2751-2758.

Blomberg, S.P., Garland, T., 2002. Tempo and mode in evolution: phylogenetic inertia, adaptation and comparative methods. J. Evol. Biol. 15, 899-910.

Burnham, K.P., Anderson, D.R., Huyvaert, K.P., 2011. AIC model selection and multimodel inference in behavioral ecology: some background, observations, and comparisons. Behav. Ecol. Sociobiol. 65, 2335.

Burr, D.B., Martin, R.B., Schaffler, M.B., Radin, E.L., 1985. Bone remodeling in response to in vivo fatigue microdamage. J. Biomech. 18, 189-200.

Butler, M.A., King, A.A., 2004. Phylogenetic comparative analysis: a modeling approach for adaptive evolution. Am. Nat. 164, 683-695.

Cardoso, L., Herman, B.C., Verborgt, O., Laudier, D., Majeska, R.J., Schaffler, M.B., 2009. Osteocyte apoptosis controls activation of intracortical resorption in response to bone fatigue. J. Bone Min. Res. 24, 597605 .

Close, R.A., Rayfield, E.J., 2012. Functional morphometric analysis of the furcula in Mesozoic birds. PloS One 7, e36664.

Currey, J., 2001. Ontogenetic changes in compact bone material properties. In: Cowin, S. (Ed.), Bone Mechanics Handbook. CRC Press, Boca Raton, pp. 19-1-19-16.

Currey, J., 2003. The many adaptations of bone. J. Biomech. 36, 1487-1495.

Diniz-Filho, J., Bini, L.M., Rangel, T.F., Morales-Castilla, I., Olalla-Tárraga, M.Á., Rodríguez, M.Á., Hawkins, B.A., 2012. On the selection of phylogenetic eigenvectors for ecological analyses. Ecography 35, 239249.

Enlow, D.H., Brown, S.O., 1957. A comparative histological study of fossil and recent bone tissues. Part II. Texas J. Sci. 9, 186-214.

Enlow, D.H., Brown, S.O., 1958. A comparative histological study of fossil and recent bone tissues. Part III. Texas J. Sci. 10, 187-230.

Ericksen, M.F., 1991. Histologic estimation of age at death using the anterior cortex of the femur. Am. J. Phys. Anthropol. 84, 171-79.

Felsenstein, J., 1973. Maximum likelihood and minimum-steps methods for estimating evolutionary trees from data on discrete characters. Syst. Zool. 22, 240-249.

Felsenstein, J., 1985. Phylogenies and the comparative method. Am. Nat. 125, 1-15.

Foote, J., 1916. A contribution to the comparative histology of the femur. Smithson. Contrib. Knowl., Vol. 35, No. 3.

Freckleton, R.P., 2009. The seven deadly sins of comparative analysis. J. Evol. Biol. 22, 1367-1375. 
Freckleton, R.P., Harvey P.H., Pagel, M., 2002. Phylogenetic analysis and comparative data: a test and review of evidence. Am. Nat. 160, 712-726.

Freckleton, R.P., Cooper, N., Jetz, W., 2011. Comparative methods as a statistical fix: the dangers of ignoring an evolutionary model. Am. Nat. 178, E10-E17.

Frost, H.M., 1987. Secondary osteon populations: an algorithm for determining mean bone tissue age. Am. J. Phys. Anthropol. 30, 221-238.

Garamszegi, L.Z. (Ed.), 2014. Modern Phylogenetic Comparative Methods and Their Application in Evolutionary Biology. Springer, Berlin, Heidelberg.

Garland, T., Midford, P.E., Ives, A.R., 1999. An introduction to phylogenetically based statistical methods, with a new method for confidence intervals on ancestral values. Am. Zool. 39, 374-388.

Grafen, A., 1989. The phylogenetic regression. Phil. Trans. R. Soc. London, Ser. B. 326, 119-157.

Gross, J., Ligges, U., 2015. nortest: Tests for Normality. R package version 1.0-4. https:/CRAN.Rproject.org/package $=$ nortest.

Harmon, L.J., Weir, J.T., Brock, C.D., Glor, R.E., Challenger, W., 2008. GEIGER: investigating evolutionary radiations. Bioinformatics 24, 129-131.

Harmon, L.J., Losos, J.B., Davies, T.J., Gillespie, R.G., Gittleman, J.L., Jennings, W.B., Kozak, K.H., McPeek, M.A., Moreno-Roark, F., Near, T.J., Purvis, A., Ricklefs, R.E., Schluter, D., Schulte II, J.A., Seehausen, O., Sidlauskas, B.L., Torres-Carvajal, O., Weir, J.T., Mooers, A.Ø., 2010. Early bursts of body size and shape evolution are rare in comparative data. Evolution 64, 2385-2396.

Hui, C., 2002. Avian furcula morphology may indicate relationships of flight requirements among birds. J. Morphol. 251, 284-293.

Jenkins, F.A., Dial, K.P. Jr., Goslow, G.E. Jr., 1988. A cineradiographic analysis of bird flight: the wishbone in starlings is a spring. Science $2411,495-1498$.

Jonsson, K.A., Fjeldsa, J., 2006. A phylogenetic supertree of oscine passerine birds (Aves: Passeri). Zool. Scripta 35, 149-186.

Kerley, E.R., 1965. The microscopic determination of age in human bone. Am. J. Phys. Anthropol. 23, 149-163.

Lee, T.C., Staines, A., Taylor, D., 2002. Bone adaptation to load: microdamage as a stimulus for bone remodelling. J. Anat. 201, 437-446.

Mason, M.W., Skedros, J.G., Bloebaum, R.D., 1995. Evidence of strain-mode-related cortical adaptation in the diaphysis of the horse radius. Bone 17, 229-237.

Mayya, A., Banerjee, A., Rajesh, R., 2013. Mammalian cortical bone in tension is non-Haversian. Sci. Rep. 3. 2533.

Mayya, A., Banerjee, A., Rajesh, R., 2016. Haversian microstructure in bovine femoral cortices: an adaptation for improved compressive strength. Mat. Sci. Eng. C 59, 454-463.

Meister, W., 1951. Changes in histological structure of the long bones of birds during the molt. Anat. Rec. 111, $1-21$.

Mori, S., Burr, D., 1993. Increased intracortical remodeling following fatigue damage. Bone 14, 103-109.

Moyle, D.D., Walker, M.W., 1986. The effects of a calcium deficient diet on the mechanical properties and morphology of goose bone. J. Biomech 19, 613-625. 
Mundry, R., 2014. Statistical issues and assumptions of phylogenetic generalized least squares. In: Garamszegi, L.Z. (Ed.), Modern Phylogenetic Comparative Methods and Their Application in Evolutionary Biology. Springer, Berlin, Heidelberg, pp. 131-156.

Münkemüller, T., Lavergne, S., Bzeznik, B., Dray, S., Jombart, T., Schiffers, K., Thuiller, W., 2012. How to measure and test phylogenetic signal. Meth. Ecol. Evol. 3, 743-756.

Orme, D., Freckleton, R., Thomas, G., Petzoldt, T., Fritz, S., Isaac, N., Pearse, W., 2013. Caper: Comparative Analyses of Phylogenetics and Evolution in R. R package version 0.5.2. https://CRAN.Rproject.org/package=caper.

Paradis, E., Claude, J., Strimmer, K., 2004. APE: analyses of phylogenetics and evolution in R language. Bioinformatics, 20, 289-290.

Parfitt, A.M., 1994. Osteonal and hemi-osteonal remodeling: the spatial and temporal framework for signal traffic in adult human bone. J. Cell. Biochem. 55, 273-286.

Parfitt, A.M., 2010. Skeletal heterogeneity and the purposes of bone remodeling: implications for the understanding of osteoporosis. In: Marcus, R., Feldman, D., Nelson, D., Rosen, C. (Eds.), Fundamentals of Osteoporosis. Academic Press, Burlington, pp. 35-52.

Pinheiro, J., Bates, D., DebRoy, S., Sarkar, D., R Core Team, 2016. nlme: Linear and Nonlinear Mixed Effects Models. R package version 3.1-126, http://CRAN.R-project.org/package=nlme.

Ponton, F., Montes, L., Castanet, J., Cubo, J., 2007. Bone histological correlates of high-frequency flapping flight and body mass in the furculae of birds: a phylogenetic approach. Biol. J. Linn. Soc. 91, 729-738.

Prum, R.O., Berv, J.S., Dornburg, A., Field, D.J., Townsend, J.P., Lemmon, E.M., Lemmon, A.R., 2015. A comprehensive phylogeny of birds (Aves) using targeted next-generation DNA sequencing. Nature 526, 569-573.

R Core Team, 2015. R: A Language and Environment for Statistical Computing. R Foundation for Statistical Computing, Vienna, Austria, https://www.R-project.org/.

Reilly, G.C, Currey, J., 1999. The development of microcracking and failure in bone depends on the loading mode to which it is adapted. J. Exp. Biol. 202, 543-552.

Robling, A.G., Castillo, A.B., Turner, C.H., 2006. Biomechanical and molecular regulation of bone remodeling. Annu. Rev. Biomed. Eng. 8, 455-498.

Saito, M., Marumo, K., 2010. Collagen cross-links as a determinant of bone quality: a possible explanation for bone fragility in aging, osteoporosis, and diabetes mellitus. Osteoporosis Int. 21, 195-214.

Skedros, J.G., Mason, M.W., Nelson, M.C., Bloebaum, R.D., 1996. Evidence of structural and material adaptation to specific strain features in cortical bone. Anat. Rec. 246, 47-63.

Skedros, J.G., Su, S.C., Bloebaum, R.D., 1997. Biomechanical implications of mineral content and microstructural variations in cortical bone of horse, elk, and sheep calcanei. Anat. Rec. 249, $297-316$.

Sorenson, S.M., Jenson, N.H., Skedros, J.G., 2004. Prevalence of atypical osteon characteristics may reflect adaptations in bending environments and during growth. J. Bone Min. Res. 19, S223 (Abstract only).

Thomas, G.H., Wills, M.A., Székely, T., 2004. A supertree approach to shorebird phylogeny. BMC Evol. Biol. $4,28$.

Viscor, G., Fuster, J.F., 1987. Relationships between morphological parameters in birds with different flying habits. Comp. Biochem. Physiol. A 87, 231-249. 
Zahnd, J., 1954. Sur les modifications histologique du squelette des oiseaux pendant la mue. Compt. Rend. Séances Soc. Biol. 148, 1491-1493. 


\section{Figure Captions}

Fig. 1. Time-calibrated phylogeny of the species investigated in the present study after Prum et al. (2015), Thomas et al. (2004) and Jonsson and Fjeldsa (2006).

Fig. 2. Example of how the regions of bone were measured. Red bar on the furcula silhouette indicates the region sectioned. Left: a normal transmitted light image of a furcula of Phasianus colchicus $(\mathrm{HBD}=0.485)$, right: the same image with coloured regions; $\mathrm{MA}=$ medullary area, $\mathrm{HBA}=$ Haversian bone area, $\mathrm{CA}=\mathrm{cortical}$ area. Whole bone area (WBA) is equivalent to $\mathrm{MA}+\mathrm{CA}$, and Haversian bone density (HBD) is equal to $\mathrm{HBA} / \mathrm{CA}$.

Fig. 3. Q-Q plot and normalized vs. fitted values of the dataset used for analysis.

Fig. 4. Box plots comparing the HBD and WBA of high- vs. low-frequency flapping, soaring vs. flapping and the different types of flight, after Viscor and Fuster (1987): 1 = high-frequency flapping flight, $2=$ rapid takeoff, short flights (cursorial birds), 3 = undulating flight, $4=$ soaring/gliding flight, and $5=$ standard forward flapping flight.

Fig. 5. Histological and microanatomical examples of sampled furculae. Note the size variation in the different species and the decrease in bone compactness from Larus to Vultur. The secondary osteons appear round except in the larger specimens, where they are more elongated. Abbreviations: SO, secondary osteon, MA, medullary area, OCL, outer circumferential layer, ICL, inner circumferential layer. 
Table 1. List of species investigated in the present study and their measurements. Haversian bone density is the ratio of Haversian bone area (HBA) over cortical area (CA). If more than one specimen was studied, their number is indicated in parentheses () and the standard deviation is given. Type of flight after Viscor and Fuster (1987): 1 = high frequency flapping; 2 = rapid take-off and short flights (cursorial birds); $3=$ undulating flight (power phase and gliding phase); 4 = gliding and soaring flight; $5=$ standard, sustained forward flapping flight. * Indicates that species was removed from final analysis to maintain normality.

\begin{tabular}{|c|c|c|c|c|c|c|c|}
\hline \multirow{2}{*}{$\begin{array}{c}\text { Species } \\
\text { Accipiter nisus }\end{array}$} & \multicolumn{2}{|c|}{$\begin{array}{l}\text { Whole bone area } \\
\left(\mathrm{mm}^{2}\right)\end{array}$} & \multicolumn{2}{|c|}{$\begin{array}{c}\text { Haversian bone } \\
\text { density } \\
(\mathrm{HBA} / \mathrm{CA})\end{array}$} & \multicolumn{2}{|c|}{$\begin{array}{c}\text { Cortical area } \\
\left(\mathrm{mm}^{2}\right)\end{array}$} & $\begin{array}{c}\text { Type of } \\
\text { flight }\end{array}$ \\
\hline & 2.26 & & 0.683 & & 1.530 & & 3 \\
\hline Alca torda (2) & 3.17 & \pm 0.540 & 0.565 & \pm 0.007 & 2.625 & \pm 0.412 & 1 \\
\hline Anas clypeata & 5.05 & & 0.376 & & 3.565 & & 1 \\
\hline Anas platyrhynchos (5) & 6.28 & \pm 1.017 & 0.435 & \pm 0.094 & 4.218 & \pm 0.655 & 1 \\
\hline Asio otus & 0.84 & & 0.520 & & 0.666 & & 3 \\
\hline Aythya fuligula & 3.98 & & 0.509 & & 3.203 & & 1 \\
\hline Buteo buteo & 2.68 & & 0.768 & & 2.574 & & 4 \\
\hline Calidris canutus* & 1.22 & & 0.172 & & 0.903 & & 5 \\
\hline Ciconia episcopus (2) & 11.36 & \pm 1.233 & 0.706 & \pm 0.188 & 7.577 & \pm 1.357 & 4 \\
\hline Columba palumbus & 1.84 & & 0.623 & & 1.100 & & 5 \\
\hline Emberiza citrinella & 0.20 & & 0.377 & & 0.183 & & 5 \\
\hline Falco tinnunculus & 1.56 & & 0.532 & & 1.116 & & 3 \\
\hline Fregata aquila & 11.88 & & 0.349 & & 3.968 & & 3 \\
\hline Fulica atra (2) & 1.95 & \pm 0.596 & 0.439 & \pm 0.285 & 1.509 & \pm 0.310 & 1 \\
\hline Garrulus glandarius & 0.47 & & 0.319 & & 0.429 & & 3 \\
\hline Gavia stellata (3) & 6.13 & \pm 2.930 & 0.680 & \pm 0.112 & 5.248 & \pm 2.421 & 1 \\
\hline Gypaetus barbatus (3) & 32.23 & \pm 4.868 & 0.824 & \pm 0.0802 & 19.340 & \pm 1.986 & 4 \\
\hline \multicolumn{8}{|l|}{ Haliaeetus } \\
\hline leucocephalus & 15.08 & & 0.739 & & 13.648 & & 4 \\
\hline
\end{tabular}




\begin{tabular}{|c|c|c|c|c|c|c|c|}
\hline Larus fuscus & 5.01 & & 0.610 & & 4.144 & & 4 \\
\hline Larus tridactyla & 2.92 & & 0.885 & & 2.038 & & 4 \\
\hline Leptoptilos dubius* & 54.65 & & 0.256 & & 20.415 & & 4 \\
\hline Leptoptilos javanicus & 42.28 & & 0.708 & & 9.733 & & 4 \\
\hline Pagodroma nivea* & 1.54 & & 0.245 & & 1.248 & & 3 \\
\hline Perdix perdix* & 2.08 & & 0.205 & & 1.717 & & 2 \\
\hline Phasianus colchicus & 3.49 & & 0.485 & & 2.710 & & 2 \\
\hline Pica pica & 0.83 & & 0.730 & & 0.769 & & 3 \\
\hline Podiceps auritus & 1.56 & & 0.691 & & 1.504 & & 1 \\
\hline Podiceps cristatus (3) & 3.26 & \pm 0.894 & 0.843 & \pm 0.011 & 2.961 & \pm 0.818 & 1 \\
\hline Podilymbus podiceps & 2.01 & & 0.754 & & 1.805 & & 1 \\
\hline Porphyrio porphyrio & 1.82 & & 0.562 & & 1.471 & & 1 \\
\hline Sarcoramphus papa & 34.07 & & 0.551 & & 14.219 & & 4 \\
\hline Scolopax rusticola & 1.34 & & 0.433 & & 1.225 & & 5 \\
\hline Strix aluco & 1.01 & & 0.570 & & 0.744 & & 3 \\
\hline Sturnus vulgaris* & 0.36 & & 0.142 & & 0.360 & & 5 \\
\hline Sula bassana (2) & 13.71 & \pm 4.973 & 0.805 & \pm 0.035 & 11.171 & \pm 3.964 & 4 \\
\hline Sylvia atricapilla* & 0.11 & & 0.065 & & 0.093 & & 5 \\
\hline Tringa hypoleucos & 0.57 & & 0.383 & & 0.444 & & 5 \\
\hline Turdus philomelos & 0.45 & & 0.661 & & 0.407 & & 5 \\
\hline Tyto alba & 0.82 & & 0.363 & & 0.661 & & 3 \\
\hline Uria aalge (2) & 5.16 & \pm 0.663 & 0.434 & \pm 0.454 & 4.057 & \pm 0.987 & 1 \\
\hline Vultur gryphus & 111.99 & & 0.765 & & 37.469 & & 4 \\
\hline
\end{tabular}


Table 2. Results of phylogenetic generalized least squares (PGLS) analyses. Significant $p$-values in bold $(p<0.05)$. WBA = whole bone area.

\begin{tabular}{|c|c|c|c|c|c|c|c|c|}
\hline PGLS & Estimate & $\begin{array}{c}\text { Standard } \\
\text { error }\end{array}$ & $t$-value & $p$-value & Lambda & $F$ statistic & $p$-value & $R$-squared \\
\hline \multicolumn{9}{|l|}{ Bivariate $(y=b 0+b 1 X)$} \\
\hline WBA & 0.08237 & 0.03548 & 2.3219 & 0.02655 & 0.278 & 5.391 & & 0.1144 \\
\hline Soaring vs flapping & -0.14984 & 0.04061 & -3.6896 & 0.0008043 & 0.104 & 13.61 & & 0.2706 \\
\hline High vs low frequency flapping & -0.01351 & 0.05175 & -0.2611 & 0.7956 & 0.411 & 0.06819 & & -0.02818 \\
\hline Type of flight & 0.00948 & 0.01602 & 0.5917 & 0.5581 & 0.425 & 0.3501 & & -0.01949 \\
\hline \multicolumn{9}{|l|}{ Additive model ( $y=b 0+b 1 X+b 2 z)$} \\
\hline WBA & 0.08234 & 0.03601 & 2.2866 & 0.02898 & 0.279 & 2.646 & 0.08644 & 0.08825 \\
\hline High vs low frequency flapping & -0.01132 & 0.04698 & -0.241 & 0.81108 & & & & \\
\hline WBA & 0.01059 & 0.04233 & 0.2501 & 0.8041 & 0.121 & 6.566 & 0.00408 & 0.2467 \\
\hline Soaring vs flapping & -0.14018 & 0.05577 & -2.5137 & 0.01717 & & & & \\
\hline WBA & 0.08265 & 0.03594 & 2.2997 & 0.02814 & 0.288 & 2.816 & 0.07472 & 0.09652 \\
\hline Type of flight & 0.00894 & 0.01463 & 0.6115 & 0.54522 & & & & \\
\hline \multicolumn{9}{|l|}{$\begin{array}{l}\text { Interaction model } \\
(y=b 0+b 1 X+b 2 z+b 3 x Z)\end{array}$} \\
\hline WBA & 0.09327 & 0.03276 & 2.847 & 0.007762 & 0 & 3.259 & 0.03462 & 0.1662 \\
\hline High vs low frequency flapping & 0.13491 & 0.09475 & 1.4239 & 0.164473 & & & & \\
\hline WBA: high vs low frequency & -0.29319 & 0.16527 & -1.774 & 0.085887 & & & & \\
\hline WBA & -0.01978 & 0.06895 & -0.2869 & 0.77612 & 0.137 & 4.35 & 0.01141 & 0.2281 \\
\hline Soaring vs flapping & -0.18051 & 0.09128 & -1.9775 & 0.05693 & & & & \\
\hline WBA: soaring vs flapping & 0.05088 & 0.08924 & 0.5701 & 0.57271 & & & & \\
\hline WBA & -0.17360 & 0.15105 & -1.1493 & 0.25921 & 0.022 & 3.313 & 0.03272 & 0.1695 \\
\hline Type of flight & -0.01810 & 0.02064 & -0.877 & 0.38725 & & & & \\
\hline WBA: type of flight & 0.06523 & 0.03765 & 1.7326 & 0.09312 & & & & \\
\hline
\end{tabular}


Table 3. Results of phylogenetic ANOVA.

\begin{tabular}{lcc}
\hline PhyANOVA & $\boldsymbol{F}$ value & $\boldsymbol{p}$-value \\
\hline \hline Soaring vs. flapping & 12.40382 & $\mathbf{0 . 0 0 1 3}$ \\
High vs. low frequency flapping & 0.09306 & 0.7622 \\
Type of flight & 0.40941 & 0.5267 \\
\hline
\end{tabular}




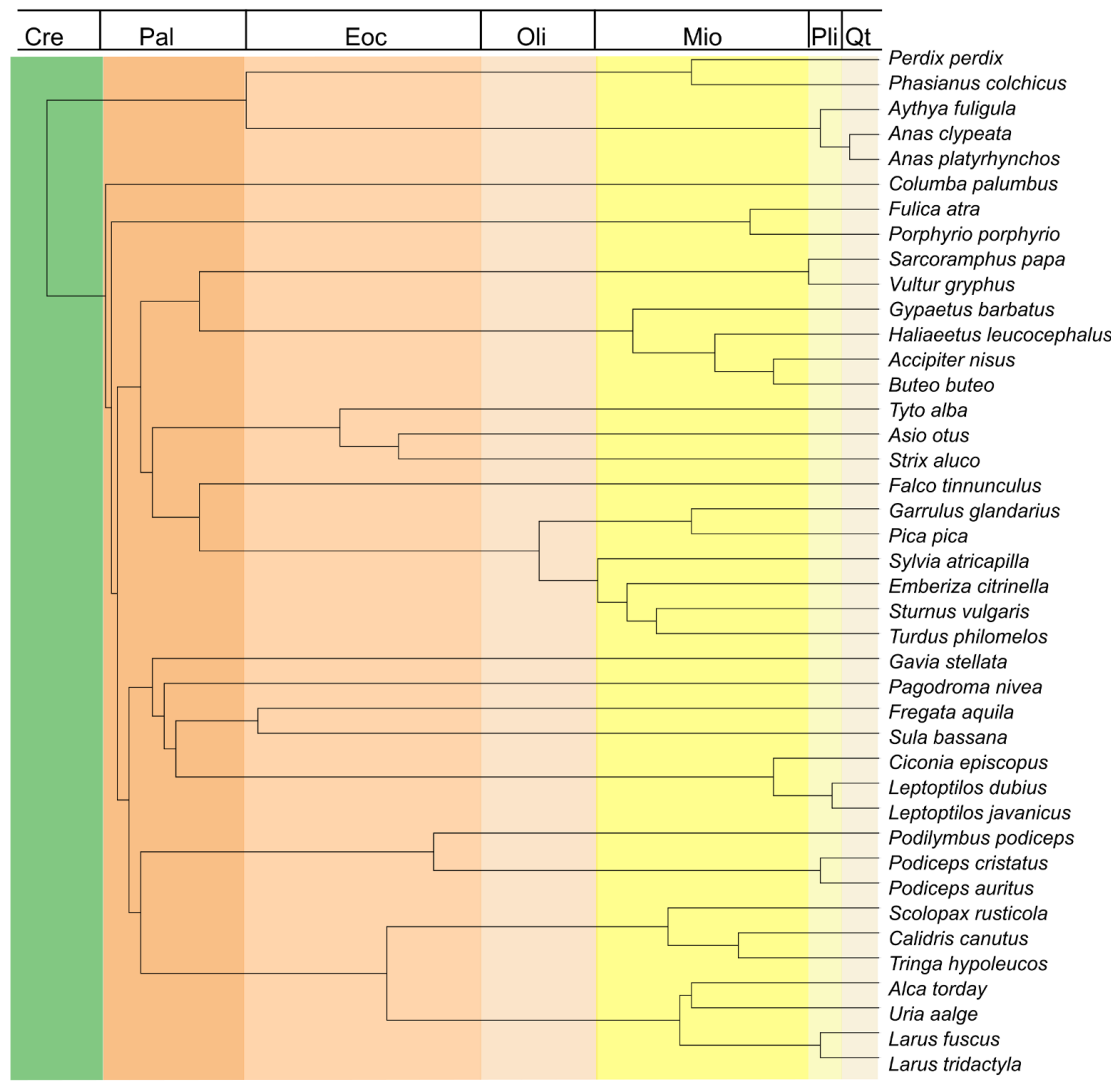

Figure 1 Time-calibrated phylogeny of the species investigated in the present study after Prum et al. (2015), Thomas et al. (2004) and Jonsson and Fjeldsa (2006).
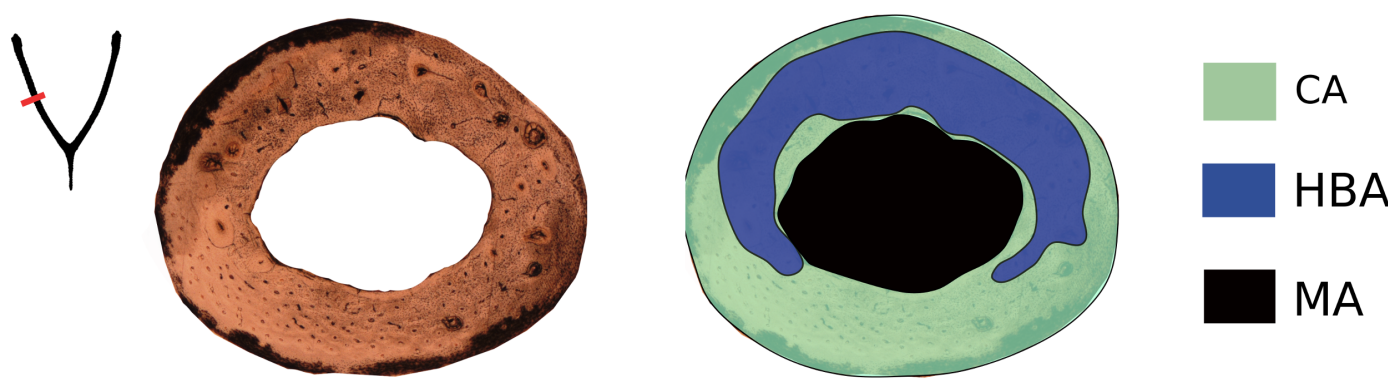

$500 \mu \mathrm{m}$

$\underline{500 \mu \mathrm{m}}$

Figure 2 Example of how the regions of bone were measured. Red bar on the furcula silhouette indicates the region sectioned. Left: a normal transmitted light image of a furcula of Phasianus colchicus $(H B D=0.485)$, right: the same image with coloured regions; $M A=$ medullary area, $H B A=$ Haversian bone area, $C A=$ cortical area. Whole bone area (WBA) is equivalent to $M A+C A$, and Haversian bone density $(H B D)$ is equal to $H B A / C A$. 
Normalized Residuals vs Fitted Values

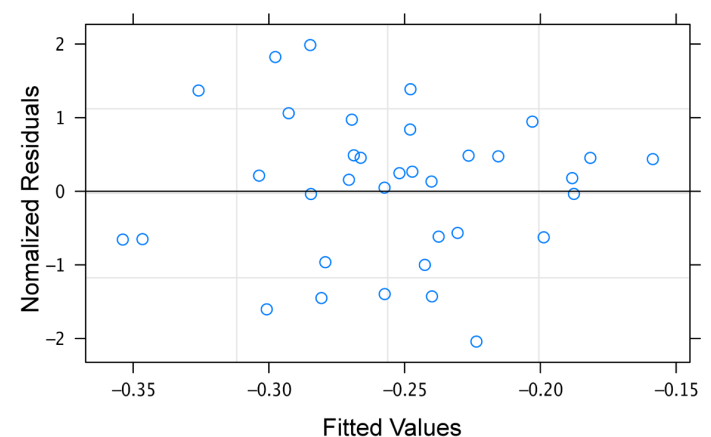

Normal Q-Q Plot

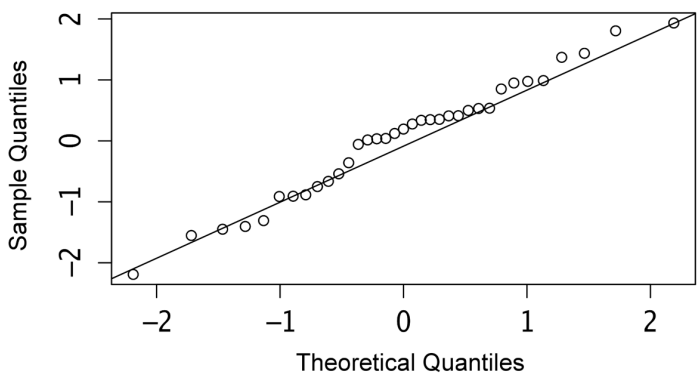

Figure $3 Q-Q$ plot and normalized vs. fitted values of the dataset used for analysis. 

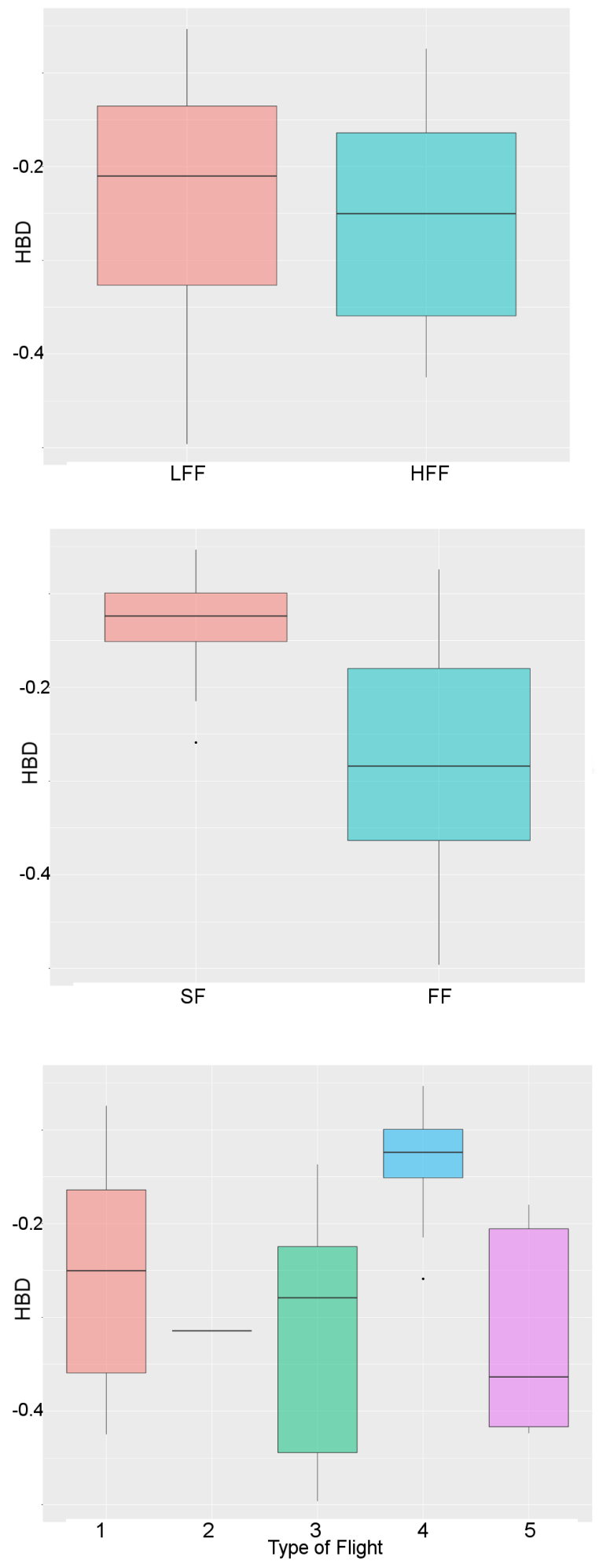

Figure 4 Box plots comparing the HBD and WBA of high-vs. low-frequency flapping (HFF, LFF), soaring vs. flapping (SF, FF) and the different types of flight, after Viscor and Fuster (1987): 1 = high-frequency flapping flight, 2 = rapid take-off, short flights (cursorial birds), 3 = undulating flight, $4=$ soaring/gliding flight, and 5 = standard forward flapping flight. 


\section{Emberiza citrinella}

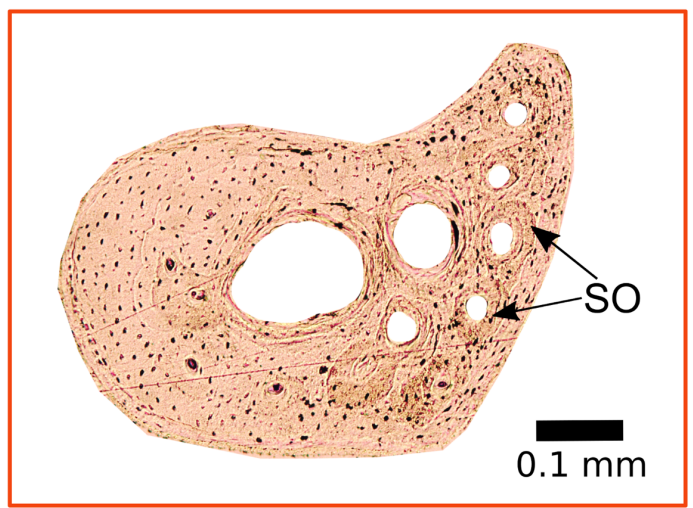

Garrulus glandarius

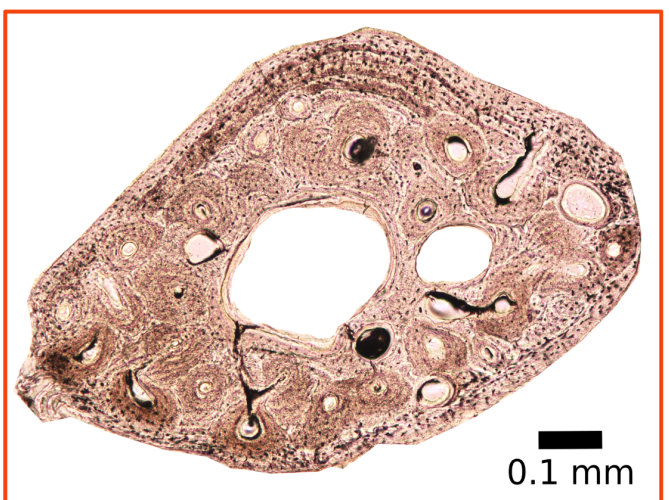

\section{Larus fuscus}
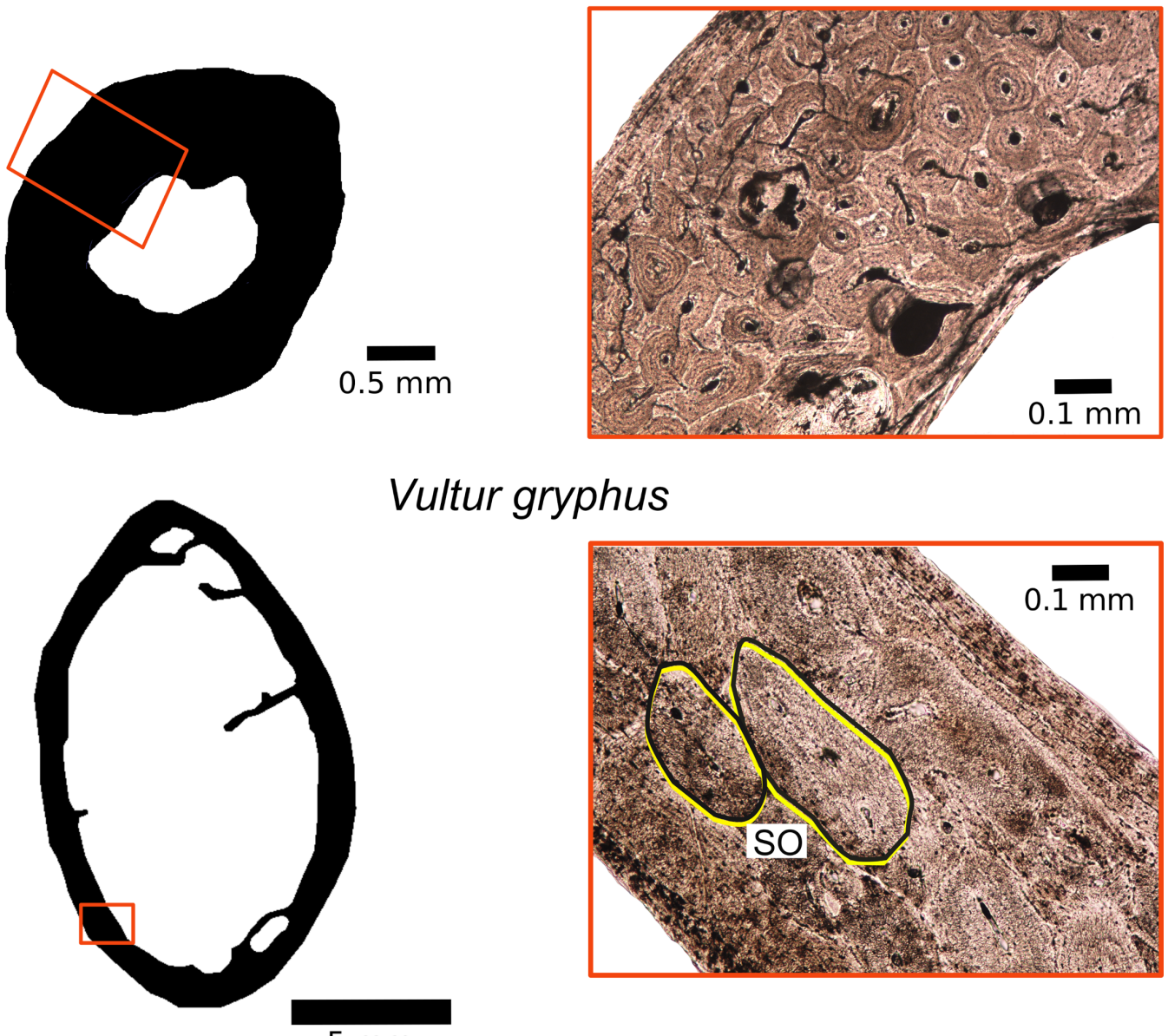

Vultur gryphus

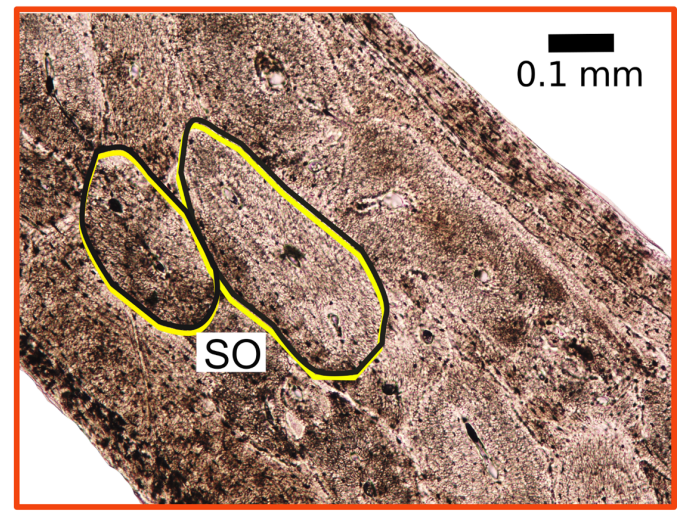

$5 \mathrm{~mm}$

Figure 5 Histological and microanatomical examples of sampled furculae. Note the size variation in the different species and the decrease in bone compactness from Larus to Vultur. The secondary osteons appear round except in the larger specimens, where they are more elongated. Abbreviations: SO, secondary osteon, MA, medullary area, OCL, outer circumferential layer, ICL, inner circumferential layer. 